\title{
Ionic liquid-accelerated Michael addition of pyrimidine and purine nucleobases to $\alpha, \beta$-unsaturated esters: a rapid approach to carboacyclic nucleosides synthesis
}

\author{
Abdolkarim Zare, ${ }^{\mathrm{a} *}$ Alireza Hasaninejad, ${ }^{\mathrm{b}}$ * Roholla Safinejad, ${ }^{\mathrm{a}}$ Ahmad Reza Moosavi- \\ Zare, ${ }^{c}$ Ali Khalafi-Nezhad, ${ }^{\mathrm{c}}$ Mohammad Hassan Beyzavi, ${ }^{\mathrm{c}}$ Mojtaba Miralai-Moredi, \\ Esmail Dehghani, ${ }^{a}$ and Parvin Kazerooni-Mojarrad ${ }^{a}$ \\ ${ }^{a}$ Department of Chemistry, Payame Noor University (PNU), Bushehr 1698, Iran \\ ${ }^{b}$ Department of Chemistry, Faculty of Sciences, Persian Gulf University, Bushehr 75169, Iran \\ ${ }^{c}$ Department of Chemistry, College of Sciences, Shiraz University, Shiraz 71454, Iran \\ E-mail:abdolkarimzare@yahoo.com, ahassaninejad@yahoo.com
}

\begin{abstract}
Ionic liquid 1-butyl-3-methylimidazolium bromide ([bmim] Br) efficiently accelerates Michael addition of pyrimidine and purine nucleobases to $\alpha, \beta$-unsaturated esters in the presence of catalytic amount of $\mathrm{Cs}_{2} \mathrm{CO}_{3}$ under microwave irradiation to give carboacyclic nucleosides in good to high yields and short reaction times.
\end{abstract}

Keywords: Ionic liquid, Michael addition, nucleobase, $\mathrm{Cs}_{2} \mathrm{CO}_{3}$, carboacyclic nucleoside, microwave

\section{Introduction}

Currently, ionic liquids are the subject of considerable interest as benign reaction media in organic synthesis because of their unique properties, such as non-volatility, non-flammability, recyclability, high thermal stability and ability to dissolve a wide range of materials. During the past decade, a variety of ionic liquids have been demonstrated as efficient and practical alternatives to volatile organic solvents for many important organic reactions, including carboncarbon, carbon-oxygen, carbon-sulfur carbon-nitrogen and carbon-phosphorus bonds formation. ${ }^{1}$

Carboacyclic nucleosides have attracted much interest due to their potential use as antiviral, ${ }^{2}$ anticancer, ${ }^{3}$ antibiotic, ${ }^{4}$ and antipsychotic agents. ${ }^{5}$ The aza-Michael addition of nucleobases to electrophilic multiple bonds has been used as a useful route toward carboacyclic nucleosides synthesis. ${ }^{6-13}$ Several catalysts have been applied to achieve this transformation, including $\mathrm{ZnO}-$ tetrabutylammonium bromide, ${ }^{6}$ 1,4-diazabicyclo[2,2,2] octane, ${ }^{7} t$-BuOK, ${ }^{8} \mathrm{NaOEt}^{9}{ } \mathrm{PBu}_{3},{ }^{10}$ enzyme, ${ }^{11}$ and $[\mathrm{bmim}] \mathrm{OH} .{ }^{12} \mathrm{~K}_{2} \mathrm{CO}_{3}$ in DMF has been also used for Michael addition of only purine nucleobases to $\alpha, \beta$-unsaturated esters as well as acrylonitrile. ${ }^{13}$ It is worth noting that the 
methods that have been established for the Michael reaction of nucleobases are associated with some drawbacks, such as long reaction time, low yield, low selectivity, the use of expensive catalysts, the use of only unhindered electrophilic alkenes, and no compliance with the green chemistry protocols. Moreover, some methods can be used for Michael reaction of only pyrimidines or only purines.

Cesium carbonate is a commercially available, heterogeneous, reusable and environmentally benign basic reagent that has been used in various organic transformations, such as $N 3$-alkylation of $N 1$-substituted pyrimidine nucleobases, ${ }^{14} \mathrm{~N}$-arylation of nucleobases, ${ }^{15} \mathrm{~N}$-alkylation of diethyl acetamidomalonate, ${ }^{16} \mathrm{O}$-alkylation of alcohols, ${ }^{17}$ diarylation of ketones, ${ }^{18} \mathrm{~S}$-alkylation of thiols, ${ }^{19}$ synthesis of calix[8]crowns by direct alkylation of $p$-tert-butylcalix[8]arene, ${ }^{20}$ and synthesis of nucleoside antibiotic Ascamycin. ${ }^{21}$

The application of microwave technology in organic synthesis has been explored extensively within the last decade. Microwave irradiation has been demonstrated to be as an efficient technique for various organic reactions instead of using conventional heating. ${ }^{21}$ This technique often leads to a remarkable decrease in reaction times, increased yields, easier workup, matches with the green chemistry protocols, and may enhance the regio- and stereoselectivity of reactions. $^{22}$

Considering the above facts, and in continuation of our previous studies on nucleoside chemistry, ${ }^{6,7,14,15,23}$ we report here an efficient green method for the synthesis of $N$-alkyl nucleobases (carboacyclic nucleosides) via microwave-assisted Michael addition of pyrimidine and purine nucleobases to $\alpha, \beta$-unsaturated esters using catalytic amount of $\mathrm{Cs}_{2} \mathrm{CO}_{3}$ in $[\mathrm{bmim}] \mathrm{Br}$ (Scheme 1).
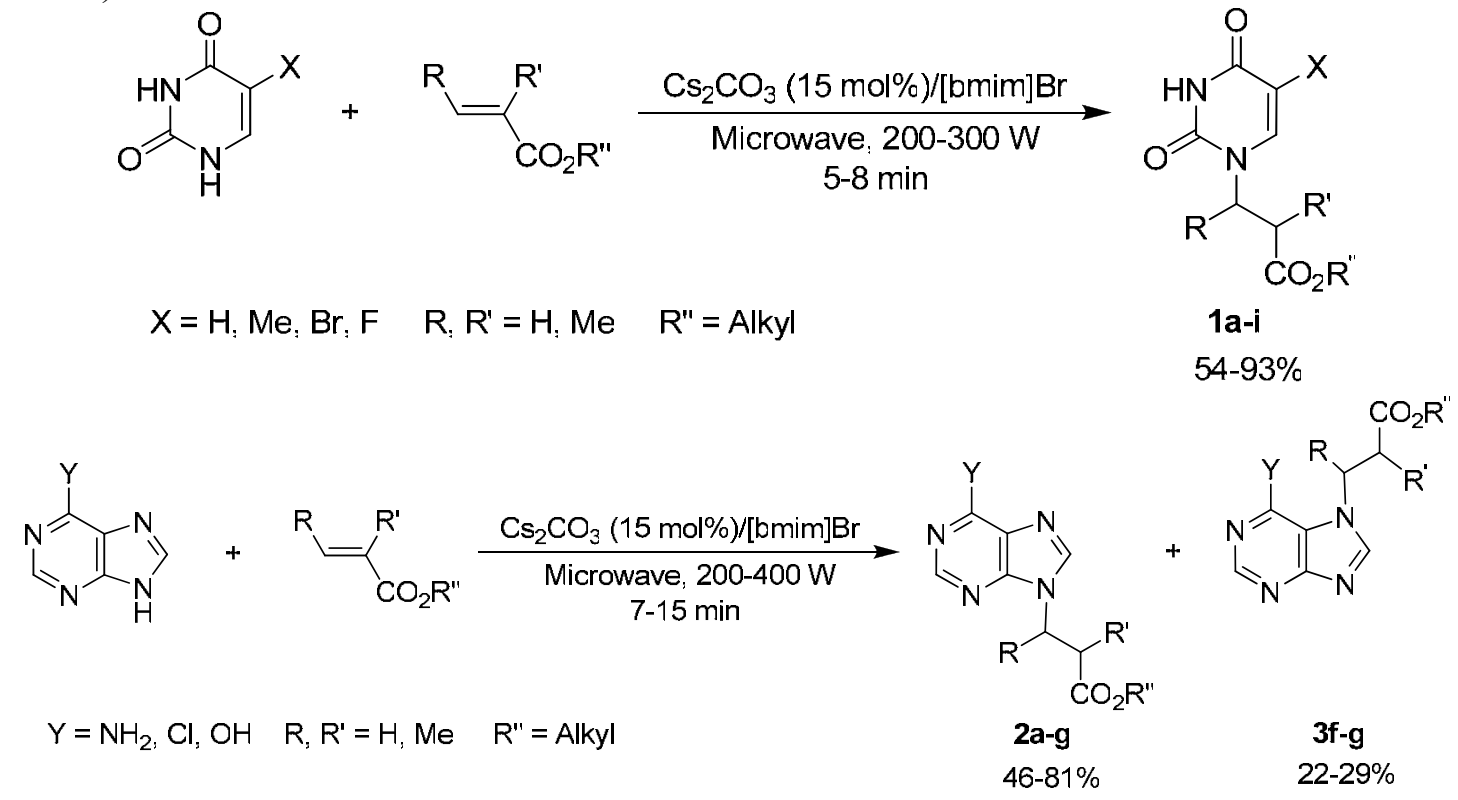

Scheme 1. Michael addition of pyrimidine and purine nucleobases to $\alpha, \beta$-unsaturated esters. 


\section{Results and Discussion}

We have found previously $\mathrm{Cs}_{2} \mathrm{CO}_{3}$ acts as an efficient basic reagent for N3-alkylation of N1substituted pyrimidines, ${ }^{14}$ and $N$-arylation of nucleobases. ${ }^{15}$ Moreover, this base has been frequently applied for alkylation reactions. ${ }^{16-21}$ These subjects encouraged us to use this base as catalyst for $\mathrm{N}$-alkylation of nucleobases via Michael addition reaction. Therefore, firstly we used different amounts of $\mathrm{Cs}_{2} \mathrm{CO}_{3}$ to accomplish Michael addition of uracil $(2 \mathrm{mmol})$ to $n$-butyl acrylate $(2.1 \mathrm{mmol})$ in $[\mathrm{bmim}] \mathrm{Br}(0.5 \mathrm{~g})$ under microwave irradiation $\left(200 \mathrm{~W}\right.$, max. $\left.110^{\circ} \mathrm{C}\right)$ to provide compound $\mathbf{1 b}$ (Scheme 1). The results are summarized in Table 1. As it can be seen from Table 1, the reaction proceeded efficiently in the presence of $15 \mathrm{~mol} \%$ of $\mathrm{Cs}_{2} \mathrm{CO}_{3}$ at $200 \mathrm{~W}$ of microwave power and the desired Michael adduct was obtained in $93 \%$ yield after 5 min. We also examined the Michael reaction in the presence of other basic catalysts (Table 1). The results showed that the catalysts $\mathrm{K}_{2} \mathrm{CO}_{3}$, basic $\mathrm{Al}_{2} \mathrm{O}_{3}, \mathrm{CaO}$ and $\mathrm{MgO}$ afforded lower yields and longer reaction times in comparison to $\mathrm{Cs}_{2} \mathrm{CO}_{3}$. Furthermore, we extended this reaction to adenine as a purine nucleobase that gave carboacyclic nucleoside $\mathbf{2 b}$ at $200 \mathrm{~W}$ of microwave power (max. $140{ }^{\circ} \mathrm{C}$ ) in $81 \%$ yield within 8 min (Scheme 1). Considering the excellent results obtained from $\mathrm{Cs}_{2} \mathrm{CO}_{3}$, we applied this catalyst for all other reactions.

Table 1. The influence of different molar ratios of $\mathrm{Cs}_{2} \mathrm{CO}_{3}$ to substrate as well as other basic catalysts on the reaction of uracil with $n$-butyl acrylate in [bmim] $\mathrm{Br}$ under microwave irradiation

\begin{tabular}{llll}
\hline Entry & Catalyst (mol\%) & Time (min) & Yield $^{\mathrm{a}}(\%)$ \\
\hline 1 & $\mathrm{Cs}_{2} \mathrm{CO}_{3}(10)$ & 7 & 88 \\
2 & $\mathrm{Cs}_{2} \mathrm{CO}_{3}(15)$ & 5 & 93 \\
3 & $\mathrm{Cs}_{2} \mathrm{CO}_{3}(20)$ & 5 & 90 \\
4 & $\mathrm{Cs}_{2} \mathrm{CO}_{3}(35)$ & 4 & 84 \\
5 & $\mathrm{~K}_{2} \mathrm{CO}_{3}(15)$ & 8 & 79 \\
6 & $\mathrm{Basic} \mathrm{Al}_{2} \mathrm{O}_{3}(15)$ & 15 & 54 \\
7 & $\mathrm{CaO}(15)$ & 15 & 48 \\
8 & $\mathrm{MgO}(15)$ & 20 & 32 \\
\hline
\end{tabular}

${ }^{\mathrm{a}}$ Isolated yield.

We also investigated the Michael reaction between uracil and $n$-butyl acrylate using $\mathrm{Cs}_{2} \mathrm{CO}_{3}$ in several ionic liquids, including $[\mathrm{bmim}] \mathrm{Br},[\mathrm{bmim}] \mathrm{Cl}$, $[\mathrm{bmim}] \mathrm{BF}_{4}$ and $[\mathrm{bmim}] \mathrm{PF}_{6}$ under microwave irradiation $\left(200 \mathrm{~W}\right.$, max. $\left.110{ }^{\circ} \mathrm{C}\right)$ (Table 2). As Table 2 indicates, higher yields and shorter reaction times were obtained in $[\mathrm{bmim}] \mathrm{Br}$ and $[\mathrm{bmim}] \mathrm{Cl}$. However, $[\mathrm{bmim}] \mathrm{Br}$ was applied as solvent for all reactions, because, the preparation of this ionic liquid was easier in comparison with the other. 
Table 2. The Michael addition of uracil to $n$-butyl acrylate using $\mathrm{Cs}_{2} \mathrm{CO}_{3}$ in different ionic liquids promoted by microwave irradiation

\begin{tabular}{llll}
\hline Entry & Ionic Liquid & Time $(\mathrm{min})$ & Yield $^{\mathrm{a}}(\%)$ \\
\hline 1 & {$[\mathrm{bmim}] \mathrm{Br}$} & 5 & 93 \\
2 & {$[\mathrm{bmim}] \mathrm{Cl}$} & 5 & 86 \\
3 & {$\left[\mathrm{bmim}^{\mathrm{B}} \mathrm{BF}_{4}\right.$} & 7 & 69 \\
4 & {$[\mathrm{bmim}] \mathrm{PF}_{6}$} & 7 & 73 \\
\hline
\end{tabular}

${ }^{\mathrm{a}}$ Isolated yield.

To compare the efficiency of ionic liquid versus the conventional solvents, we examined the model reaction in some conventional solvents. Thus, a mixture of uracil $(2 \mathrm{mmol}), \mathrm{Cs}_{2} \mathrm{CO}_{3}(15$ mol\%) and $n$-butyl acrylate $(2.1 \mathrm{mmol})$ was irradiated in a microwave oven $(200 \mathrm{~W}$, max. $110^{\circ} \mathrm{C}$ ) in several conventional solvents $(5 \mathrm{~mL})$ (Table 3). As it is shown in Table 3, higher yield and shorter reaction time were observed in $[\mathrm{bmim}] \mathrm{Br}$ with respect to the conventional solvents. Therefore, ionic liquid is an essential factor to promote the Michael reaction.

Table 3. Comparative the Michael addition of uracil to $n$-butyl acrylate using $\mathrm{Cs}_{2} \mathrm{CO}_{3}$ in conventional solvents versus $[\mathrm{bmim}] \mathrm{Br}$ under microwave irradiation $\left(200 \mathrm{~W}\right.$, max. $\left.110^{\circ} \mathrm{C}\right)$

\begin{tabular}{llll}
\hline Entry & Solvent & Time $(\mathrm{min})$ & Yield $^{\mathrm{a}}(\%)$ \\
\hline 1 & {$[$ bmim]Br } & 5 & 93 \\
2 & DMF & 17 & 54 \\
3 & DMSO & 12 & 62 \\
4 & o-Xylene & 20 & 21 \\
5 & Solvent-free & 20 & 17 \\
\hline
\end{tabular}

${ }^{\mathrm{a}}$ Isolated yield.

We also studied the effectiveness of microwave heating in comparison to conventional heating on the Michael reaction. For this purpose, compounds $\mathbf{1 b}$ as well as $\mathbf{2 b}$ were also prepared via Michael addition of uracil as well as adenine to $n$-butyl acrylate in the presence of $\mathrm{Cs}_{2} \mathrm{CO}_{3}$ in $[\mathrm{bmim}] \mathrm{Br}$ under thermal conditions. The results are displayed in Table 4. Clearly, the microwave procedure is more efficient. It must be mentioned that increasing the reaction temperature or the reaction times under the conventional heating did not improve the reaction yields. 
Table 4. Comparative synthesis of compounds $\mathbf{1 b}$ and $\mathbf{2 b}$ using conventional heating versus the microwave method $(200 \mathrm{~W})$

\begin{tabular}{llllll}
\hline \multirow{2}{*}{ Compound } & \multirow{2}{*}{ Temperature $^{\mathrm{a}}\left({ }^{\circ} \mathrm{C}\right)$} & \multicolumn{2}{l}{ Conventional Heating } & \multicolumn{2}{l}{ Microwave Irradiation } \\
\cline { 3 - 6 } & & Time $(\min )$ & Yield $^{\mathrm{b}}(\%)$ & Time $(\mathrm{min})$ & Yield $^{\mathrm{b}}(\%)$ \\
\hline $\mathbf{1 b}$ & 110 & 240 & 54 & 5 & 93 \\
$\mathbf{2 b}$ & 140 & 360 & 46 & 8 & 81 \\
\hline
\end{tabular}

${ }^{\mathrm{a}}$ Temperature in microwave and thermal conditions. ${ }^{\mathrm{b}}$ Isolated yield.

To realize the generality and scope of this method, different pyrimidine and purine nucleobases were introduced to structurally diverse $\alpha, \beta$-unsaturated esters (Table 5 ). As Table 5 indicates, all reactions proceeded efficiently and the desired Michael adducts were obtained in good to high yields and short reaction times. It has been observed that the bulkiness of alkoxy group (-OR) in the $\alpha, \beta$-unsaturated esters affected slightly on the Michael reactions. The bulkier alkoxy groups afforded lower yields and longer reaction times (Table 5, entries 1-4 and 10-12). The structure of $\alpha, \beta$-unsaturated esters had significant effect on the reaction. When nucleobases were reacted with sterically hindered $\alpha, \beta$-unsaturated esters such as ethyl methacrylate and ethyl crotonate, the reaction yields decreased (Table 5, entries 5, 6, 13 and 14). Moreover, higher microwave power was applied in these cases. The presence of substituents ( $\mathrm{Me}, \mathrm{Br}$ and $\mathrm{F}$ ) on 5 position of the pyrimidine nucleobases had no significant effect on the reaction results (Table 5, entries 7-9). When adenine was used in the Michael reaction, exclusively N9-alkylated products were produced (Table 5, entries 10-14). However, Michael reactions of 6-chloropurine and hypoxanthine gave $N 7$-isomers beside $N 9$-ones in low yields (Table 5 , entries 15 and 16 ).

Table 5. Michael addition of pyrimidine and purine nucleobases to $\alpha, \beta$-unsaturated esters in the presence of $\mathrm{Cs}_{2} \mathrm{CO}_{3}$ in [bmim] $\mathrm{Br}$ promoted by microwave irradiation

\begin{tabular}{llcccc}
\hline Entry & MW Power $(\mathrm{W})$ & $\mathrm{T}\left({ }^{\circ} \mathrm{C}\right)$ & Time $(\mathrm{min})$ & Yield $^{\mathrm{a}}(\%)$ \\
\hline $1^{\mathrm{b}}$ & & & & \\
\hline
\end{tabular}


Table 5. Continued

\begin{tabular}{|c|c|c|c|c|c|}
\hline Entry & Product & MW Power (W) & $\mathrm{T}\left({ }^{\mathrm{o}} \mathrm{C}\right)$ & Time (min) & Yield $^{\mathrm{a}}(\%)$ \\
\hline $3^{c}$ & & 200 & 110 & 6 & 87 \\
\hline $4^{c}$ & & 200 & 110 & 8 & 84 \\
\hline $5^{b}$ & & 300 & 130 & 5 & 67 \\
\hline $6^{\mathrm{b}}$ & & 300 & 140 & 6 & 54 \\
\hline $7^{b}$ & & 200 & 110 & 5 & 87 \\
\hline $8^{b}$ & & 200 & 110 & 5 & 88 \\
\hline$P 9^{b}$ & & 200 & 110 & 5 & 91 \\
\hline
\end{tabular}


Table 5. Continued

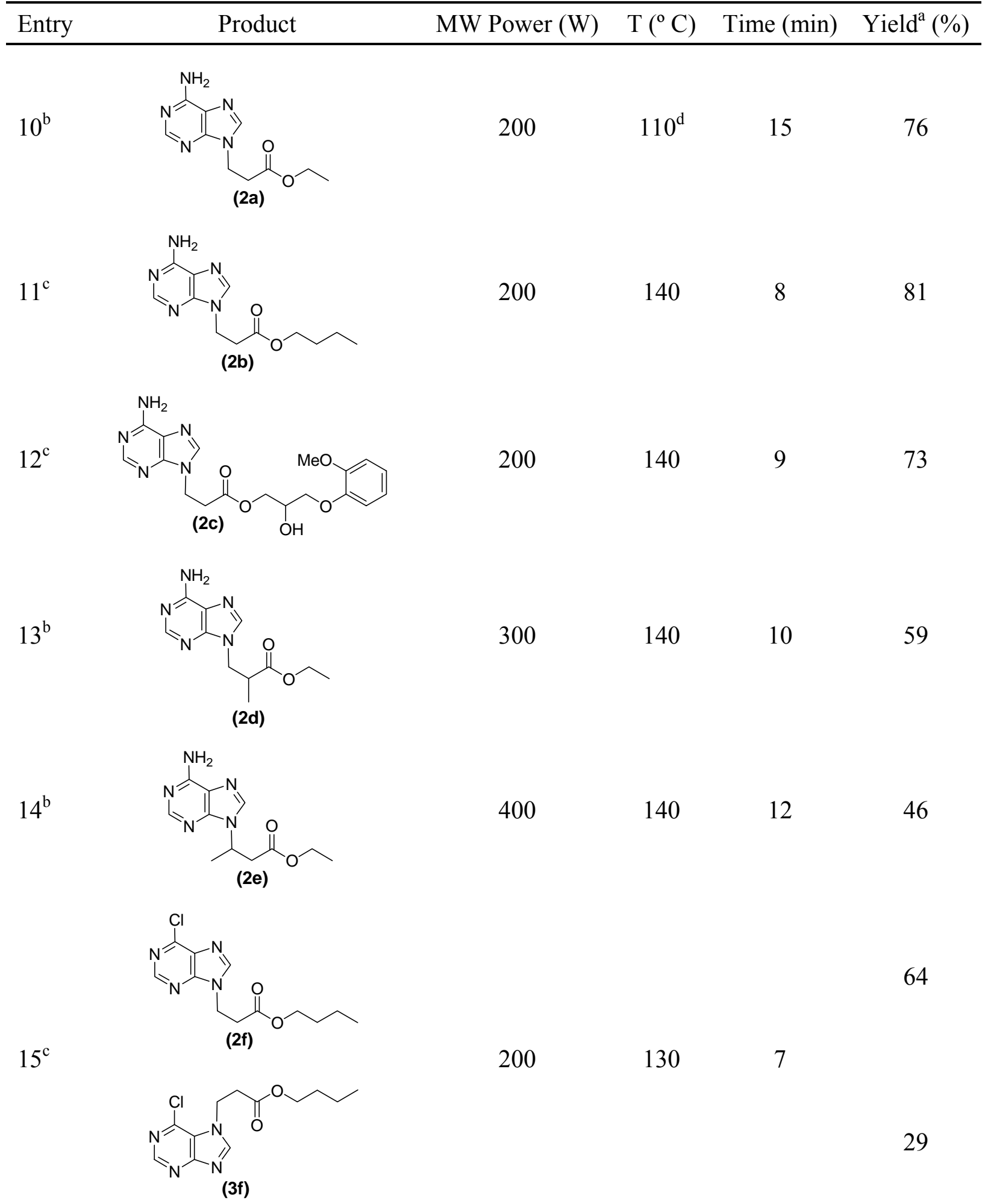


Table 5. Continued

\begin{tabular}{lllll}
\hline Entry & MW Power $(\mathrm{W})$ & $\mathrm{T}\left({ }^{\mathrm{o}} \mathrm{C}\right)$ & Time $(\mathrm{min})$ & Yield $^{\mathrm{a}}(\%)$ \\
\hline $16^{\mathrm{c}}$ & & & & \\
\hline
\end{tabular}

${ }^{\mathrm{a}}$ Isolated yield. ${ }^{\mathrm{b}} \mathrm{In}$ this reaction, the ester/nucleobase ratio $(\mathrm{mol} / \mathrm{mol})$ was $1.15 / 1 .{ }^{\mathrm{c}} \mathrm{In}$ this reaction, the ester/nucleobase ratio $(\mathrm{mol} / \mathrm{mol})$ was $1.05 / 1$. ${ }^{\mathrm{d}}$ This reaction was performed at $110^{\circ} \mathrm{C}$ because of lower boiling point of the corresponding $\alpha, \beta$-unsaturated ester, ethyl acrylate.

The interesting behavior of $[\mathrm{bmim}] \mathrm{Br} / \mathrm{Cs}_{2} \mathrm{CO}_{3}$ system lies in the fact that it can be re-used after simple washing with $\mathrm{Et}_{2} \mathrm{O}$, rendering thus process more economical. The yields of compound $\mathbf{1 b}$ (model compound) in the $2^{\text {nd }}$ and $3^{\text {rd }}$ uses of the $[\mathrm{bmim}] \mathrm{Br} / \mathrm{Cs}_{2} \mathrm{CO}_{3}$ were almost as high as in the first use.

To compare the efficiency and capacity of the present method with the reported methods for the Michael reaction of nucleobases, we have tabulated the results of some these methods in the synthesis of carboacyclic nucleosides $\mathbf{1 b}, \mathbf{1 e}, \mathbf{1 f}, \mathbf{2 b}, \mathbf{2 d}$ and $\mathbf{2 e}$ in Table 6 . As it is clear from Table 6, our method have significantly improved Michael addition of pyrimidine nucleobases to sterically hindered $\alpha, \beta$-unsaturated esters as well as Michael reaction between purines with either unhindered or hindered $\alpha, \beta$-unsaturated esters.

\section{Conclusions}

In summary, Michael addition of pyrimidine and purine nucleobases to $\alpha, \beta$-unsaturated esters can be effectively performed in ionic liquids under microwave irradiation. This new method for the synthesis of carboacyclic nucleosides has the advantage of high yield, high selectivity, short reaction time, generality, ease of product isolation, potential for recycling of ionic liquid as well as catalyst, and compliance with the green chemistry protocols. 
Table 6. Comparative synthesis of compounds $\mathbf{1 b}, \mathbf{1 e}, \mathbf{1 f}, \mathbf{2 b}, \mathbf{2 d}$ and $2 \mathbf{e}$ using the reported methods versus our method

\begin{tabular}{|c|c|c|c|c|c|c|c|c|}
\hline \multirow[b]{2}{*}{ Compound } & \multicolumn{2}{|c|}{ Cai et al. ${ }^{11}$} & \multicolumn{2}{|c|}{ Khalafi-Nezhad et al. $^{7}$} & \multicolumn{2}{|c|}{ Zare et al. $^{6}$} & \multicolumn{2}{|c|}{ Present Method } \\
\hline & $\begin{array}{l}\text { Time } \\
(\min )\end{array}$ & $\begin{array}{l}\text { Yield } \\
(\%) \\
\end{array}$ & $\begin{array}{l}\text { Time } \\
(\mathrm{min})\end{array}$ & $\begin{array}{l}\text { Yield } \\
(\%)\end{array}$ & $\begin{array}{l}\text { Time } \\
(\mathrm{min})\end{array}$ & $\begin{array}{l}\text { Yield } \\
(\%)\end{array}$ & $\begin{array}{l}\text { Time } \\
(\mathrm{min})\end{array}$ & $\begin{array}{l}\text { Yield } \\
(\%)\end{array}$ \\
\hline $1 b$ & 720 & 44 & 6 & 85 & 25 & 93 & 5 & 93 \\
\hline $1 \mathrm{e}$ & - & - & 10 & 40 & 20 & 48 & 5 & 67 \\
\hline 1f & - & - & 9 & 27 & 15 & 41 & 6 & 54 \\
\hline $2 \mathbf{b}$ & - & - & 8 & 73 & 20 & 74 & 8 & 81 \\
\hline 2d & - & - & 12 & 38 & 15 & 37 & 10 & 59 \\
\hline $2 \mathrm{e}$ & - & - & 10 & 24 & 20 & 29 & 12 & 46 \\
\hline
\end{tabular}

\section{Experimental Section}

General Procedures. All chemicals were purchased from Merck or Fluka Chemical Companies. All known compounds were identified by comparison of their melting points and spectral data with those in the authentic samples. The reactions were carried out using laboratory microwave oven (MW 3000, Landgraf Company, Germany). The ${ }^{1} \mathrm{H}$ NMR (250 MHz) and ${ }^{13} \mathrm{C}$ NMR (62.5 $\mathrm{MHz}$ ) were run on a Bruker Avance DPX-250, FT-NMR spectrometer. Mass spectra were recorded on a Shimadzu GC MS-QP 1000 EX apparatus. Microanalyses were performed on a Perkin-Elmer 240-B microanalyzer. Melting points were recorded on a Büchi B-545 apparatus in open capillary tubes.

\section{General procedure for Michael addition of nucleobases to $\alpha, \beta$-unsaturated esters in ionic liquids}

To a well-ground mixture of nucleobase $(2 \mathrm{mmol})$ and $\mathrm{Cs}_{2} \mathrm{CO}_{3}(0.098 \mathrm{~g}, 0.3 \mathrm{mmol})$ in a microwave vessel was added $[\mathrm{bmim}] \mathrm{Br}(0.5 \mathrm{~g})$ and $\alpha, \beta$-unsaturated ester (2.1 to $2.3 \mathrm{mmol})$ (Table 4), and mixed carefully with a small rod. The resulting mixture was irradiated in a microwave oven for the powers, the temperatures and the times reported in Table 4. Afterward, the reaction mixture was cooled to room temperature and was extracted with $\mathrm{Et}_{2} \mathrm{O}(3 \times 50 \mathrm{~mL})$. The organic extracts were then combined. After removal of the solvent, the crude product was purified by column chromatography on silica gel eluted with EtOAc/n-hexane. After isolation of the product and evaporating of the remaining $\mathrm{Et}_{2} \mathrm{O}$ in ionic liquid, the ionic liquid containing the catalyst $\left(\mathrm{Cs}_{2} \mathrm{CO}_{3} /[\mathrm{bmim}] \mathrm{Br}\right)$ was used for next run under identical reaction conditions.

Ethyl 3-(2,4-dioxo-3,4-dihydropyrimidin-1(2H)-yl)propanoate (1a). Column chromathography on silica gel eluted with EtOAc/n-hexane (2/1) gave a colorless solid; mp 77$79{ }^{\circ} \mathrm{C}$ (Lit. $\left.{ }^{6} \mathrm{mp} 77-79{ }^{\circ} \mathrm{C}\right) ;{ }^{1} \mathrm{H}$ NMR $\left(\mathrm{CDCl}_{3}\right): \delta 1.25\left(3 \mathrm{H}, \mathrm{t}, J=7.0 \mathrm{~Hz}, \mathrm{CH}_{3}\right), 2.74(2 \mathrm{H}, \mathrm{t}, J=6.0$ 
$\left.\mathrm{Hz}, \mathrm{O}=\mathrm{CCH}_{2}\right), 3.85\left(2 \mathrm{H}, \mathrm{t}, J=6.0 \mathrm{~Hz}, \mathrm{NCH}_{2}\right), 4.11\left(2 \mathrm{H}, \mathrm{q}, J=7.0 \mathrm{~Hz}, \mathrm{OCH}_{2}\right), 5.64(1 \mathrm{H}, \mathrm{d}, J=$ $7.9 \mathrm{~Hz}, \mathrm{H}_{5}$ of uracil), $7.26\left(1 \mathrm{H}, \mathrm{d}, J=7.9 \mathrm{~Hz}, \mathrm{H}_{6}\right.$ of uracil), $10.19(1 \mathrm{H}, \mathrm{s}, \mathrm{NH})$.

Butyl 3-(2,4-dioxo-3,4-dihydropyrimidin-1(2H)-yl)propanoate (1b). Column chromathography on silica gel eluted with EtOAc/n-hexane (2/1) gave a colorless solid; mp 62$64{ }^{\circ} \mathrm{C}\left(\right.$ Lit. $\left.^{6} \mathrm{mp} \mathrm{61-63}{ }^{\circ} \mathrm{C}\right) ;{ }^{1} \mathrm{H}$ NMR $\left(\mathrm{CDCl}_{3}\right): \delta 0.92\left(3 \mathrm{H}, \mathrm{t}, J=6.8 \mathrm{~Hz}, \mathrm{CH}_{3}\right), 1.35(2 \mathrm{H}, \mathrm{m}$, $\left.\mathrm{CH}_{3} \mathrm{CH}_{2}\right), 1.56\left(2 \mathrm{H}, \mathrm{m}, \mathrm{CH}_{3} \mathrm{CH}_{2} \mathrm{CH}_{2}\right), 2.72\left(2 \mathrm{H}, \mathrm{t}, J=5.9 \mathrm{~Hz}, \mathrm{O}=\mathrm{CCH}_{2}\right), 3.80(2 \mathrm{H}, \mathrm{t}, J=5.9 \mathrm{~Hz}$, $\left.\mathrm{NCH}_{2}\right), 4.09\left(2 \mathrm{H}, \mathrm{t}, J=6.9 \mathrm{~Hz}, \mathrm{OCH}_{2}\right), 5.66\left(1 \mathrm{H}, \mathrm{d}, J=7.9 \mathrm{~Hz}, \mathrm{H}_{5}\right.$ of uracil $), 7.25(1 \mathrm{H}, \mathrm{d}, J=$ $7.9 \mathrm{~Hz}, \mathrm{H}_{6}$ of uracil), $10.22(1 \mathrm{H}, \mathrm{s}, \mathrm{NH})$.

Phenethyl 3-(2,4-dioxo-3,4-dihydropyrimidin-1(2H)-yl)propanoate $\quad$ (1c). Column chromathography on silica gel eluted with EtOAc/n-hexane (2/1) gave a pale yellow buff (Lit. ${ }^{6}$ buff); ${ }^{1} \mathrm{H} \mathrm{NMR}\left(\mathrm{CDCl}_{3}\right): \delta 2.71\left(2 \mathrm{H}, \mathrm{t}, J=5.8 \mathrm{~Hz}, \mathrm{O}=\mathrm{CCH}_{2}\right), 2.86\left(2 \mathrm{H}, \mathrm{t}, J=7.0 \mathrm{~Hz}, \mathrm{PhCH}_{2}\right)$, $3.81\left(2 \mathrm{H}, \mathrm{t}, J=5.8 \mathrm{~Hz}, \mathrm{NCH}_{2}\right), 4.32\left(2 \mathrm{H}, \mathrm{t}, J=7.0 \mathrm{~Hz}, \mathrm{OCH}_{2}\right), 5.60\left(1 \mathrm{H}, \mathrm{d}, J=7.9 \mathrm{~Hz}, \mathrm{H}_{5}\right.$ of uracil), 7.11-7.25 (6H, complex, $\mathrm{H}_{1}-\mathrm{H}_{5}$ of phenyl group and $\mathrm{H}_{6}$ of uracil $), 10.26(1 \mathrm{H}, \mathrm{s}, \mathrm{NH})$.

2-Hydroxy-3-(2-methoxyphenoxy)propyl

3-(2,4-dioxo-3,4-dihydropyrimidin-1(2H)yl)propanoate (1d). Column chromathography on silica gel eluted with EtOAc/n-hexane (2/1) gave a pale yellow oil; IR (neat): 3468, 3170, 3063, 2972, 1732, 1684, $1647 \mathrm{~cm}^{-1}$; ${ }^{1} \mathrm{H}$ NMR $\left(\mathrm{CDCl}_{3}\right): \delta 2.75\left(2 \mathrm{H}, \mathrm{t}, J=5.9 \mathrm{~Hz}, \mathrm{O}=\mathrm{CCH}_{2}\right), 3.66\left(3 \mathrm{H}, \mathrm{s}, \mathrm{CH}_{3}\right), 3.81-3.92(5 \mathrm{H}, \mathrm{m}), 4.15-4.26$ $(3 \mathrm{H}, \mathrm{m}), 5.63\left(1 \mathrm{H}, \mathrm{d}, J=8.0 \mathrm{~Hz}, \mathrm{H}_{5}\right.$ of uracil), 6.71-6.79 $(4 \mathrm{H}, \mathrm{m}), 7.20\left(1 \mathrm{H}, \mathrm{d}, J=8.0 \mathrm{~Hz}, \mathrm{H}_{6}\right.$ of uracil), $10.25(1 \mathrm{H}, \mathrm{s}, \mathrm{NH}) ;{ }^{13} \mathrm{C} \mathrm{NMR}\left(\mathrm{CDCl}_{3}\right): \delta 55.9,65.4,68.3,70.8,36.6,45.9,102.3,112.3$, 114.6, 121.2, 122.0, 145.0, 147.5, 149.7, 151.6, 164.0, 170.6; MS (m/z): $364\left(\mathrm{M}^{+}\right)$; Anal. calcd. for $\mathrm{C}_{17} \mathrm{H}_{20} \mathrm{~N}_{2} \mathrm{O}_{7}$ : C, 56.04; H, 5.53; N, 7.69. Found: C, 56.25; H, 5.70; N, 7.49.

Ethyl 3-(2,4-dioxo-3,4-dihydropyrimidin-1(2H)-yl)-2-methylpropanoate (1e). Column chromathography on silica gel eluted with EtOAc/n-hexane (2/1) gave a pale yellow oil (Lit. ${ }^{7}$ oil); ${ }^{1} \mathrm{H}$ NMR $\left(\mathrm{CDCl}_{3}\right): \delta$ 1.16-1.21 $\left(6 \mathrm{H}, \mathrm{m}, \mathrm{CH}_{2} \mathrm{CH}_{3}\right.$ and $\left.\mathrm{CHCH}_{3}\right), 2.95(1 \mathrm{H}, \mathrm{m}, \mathrm{O}=\mathrm{CCH}), 3.64$ $\left(1 \mathrm{H}, \mathrm{m}\right.$, one $\mathrm{H}$ of $\left.\mathrm{NCH}_{2}\right), 3.82\left(1 \mathrm{H}, \mathrm{m}\right.$, one $\mathrm{H}$ of $\left.\mathrm{NCH}_{2}\right), 4.12\left(2 \mathrm{H}, \mathrm{q}, J=7.0 \mathrm{~Hz}, \mathrm{OCH}_{2}\right), 5.63$ $\left(1 \mathrm{H}, \mathrm{d}, J=7.9 \mathrm{~Hz}, \mathrm{H}_{5}\right.$ of uracil), $7.22\left(1 \mathrm{H}, \mathrm{d}, J=7.9 \mathrm{~Hz}, \mathrm{H}_{6}\right.$ of uracil $), 10.22(1 \mathrm{H}, \mathrm{s}, \mathrm{NH})$.

Ethyl 3-(2,4-dioxo-3,4-dihydropyrimidin-1(2H)-yl)butanoate (1f). Column chromathography on silica gel eluted with EtOAc/n-hexane (2/1) gave a pale yellow solid; mp $115-117{ }^{\circ} \mathrm{C}$ (Lit. ${ }^{7}$ mp 116-118 $\left.{ }^{\circ} \mathrm{C}\right) ;{ }^{1} \mathrm{H} \mathrm{NMR}\left(\mathrm{CDCl}_{3}\right): \delta 1.22\left(3 \mathrm{H}, \mathrm{t}, J=7.0 \mathrm{~Hz}, \mathrm{CH}_{2} \mathrm{CH}_{3}\right), 1.39(3 \mathrm{H}, \mathrm{d}, J=6.9 \mathrm{~Hz}$, $\left.\mathrm{CHCH}_{3}\right), 2.65\left(1 \mathrm{H}, \mathrm{m}\right.$, one $\mathrm{H}$ of $\left.\mathrm{O}=\mathrm{CCH}_{2}\right), 2.86\left(1 \mathrm{H}, \mathrm{m}\right.$, one $\mathrm{H}$ of $\left.\mathrm{O}=\mathrm{CCH}_{2}\right), 4.11(2 \mathrm{H}, \mathrm{q}, J=7.0$ $\left.\mathrm{Hz}, \mathrm{OCH}_{2}\right), 4.65\left(1 \mathrm{H}, \mathrm{m}, \mathrm{CH}_{3} \mathrm{CH}\right), 5.65\left(1 \mathrm{H}, \mathrm{d}, J=7.9 \mathrm{~Hz}, \mathrm{H}_{5}\right.$ of uracil $), 7.25(1 \mathrm{H}, \mathrm{d}, J=7.9$ $\mathrm{Hz}, \mathrm{H}_{6}$ of uracil), 10.24 (1H, s, NH).

Ethyl 3-(5-methyl-2,4-dioxo-3,4-dihydropyrimidin-1(2H)-yl)propanoate (1g). Column chromathography on silica gel eluted with EtOAc/n-hexane (2/1) gave a colorless solid; mp 145$147{ }^{\circ} \mathrm{C}$ (Lit. $\left.{ }^{11} \mathrm{mp} \mathrm{149-150}{ }^{\circ} \mathrm{C}\right) ;{ }^{1} \mathrm{H} \mathrm{NMR}\left(\mathrm{CDCl}_{3}\right): \delta 1.24\left(3 \mathrm{H}, \mathrm{t}, J=7.1 \mathrm{~Hz}, \mathrm{CH}_{2} \mathrm{CH}_{3}\right), 1.88$ $\left(3 \mathrm{H}, \mathrm{s}, \mathrm{CH}_{3}\right), 2.75\left(2 \mathrm{H}, \mathrm{t}, J=6.0 \mathrm{~Hz}, \mathrm{O}=\mathrm{CCH}_{2}\right), 3.83\left(2 \mathrm{H}, \mathrm{t}, J=6.0 \mathrm{~Hz}, \mathrm{NCH}_{2}\right), 4.12(2 \mathrm{H}, \mathrm{q}, J=$ $\left.7.1 \mathrm{~Hz}, \mathrm{OCH}_{2}\right), 7.25\left(1 \mathrm{H}, \mathrm{s}, \mathrm{H}_{6}\right.$ of thymine), $10.19(1 \mathrm{H}, \mathrm{s}, \mathrm{NH})$.

Ethyl 3-(5-bromo-2,4-dioxo-3,4-dihydropyrimidin-1(2H)-yl)propanoate (1h). Column chromathography on silica gel and eluting with EtOAc/ $n$-hexane (2/1) gave a pale yellow solid; mp 139-145 ${ }^{\circ} \mathrm{C}$ (dec.) [(Lit. ${ }^{11} 143-150{ }^{\circ} \mathrm{C}$ (dec.) ]; ${ }^{1} \mathrm{H}$ NMR $\left(\mathrm{CDCl}_{3}\right): \delta 1.25(3 \mathrm{H}, \mathrm{t}, \mathrm{J}=7.0 \mathrm{~Hz}$, 
$\left.\mathrm{CH}_{3}\right), 2.79\left(2 \mathrm{H}, \mathrm{t}, J=5.8 \mathrm{~Hz}, \mathrm{O}=\mathrm{CCH}_{2}\right), 3.82\left(2 \mathrm{H}, \mathrm{t}, J=5.8 \mathrm{~Hz}, \mathrm{NCH}_{2}\right), 4.12(2 \mathrm{H}, \mathrm{q}, J=7.0 \mathrm{~Hz}$, $\left.\mathrm{OCH}_{2}\right), 7.61\left(1 \mathrm{H}, \mathrm{s}, \mathrm{H}_{6}\right.$ of 5-bromouracil), $10.27(1 \mathrm{H}, \mathrm{s}, \mathrm{NH})$.

Ethyl 3-(5-fluoro-2,4-dioxo-3,4-dihydropyrimidin-1(2H)-yl)propanoate (1i). Column chromathography on silica gel eluted with EtOAc/n-hexane (2/1) gave a colorless solid; mp 122$124{ }^{\circ} \mathrm{C}$ (Lit. $\left.{ }^{11} \mathrm{mp} 124-126{ }^{\circ} \mathrm{C}\right) ;{ }^{1} \mathrm{H}$ NMR $\left(\mathrm{CDCl}_{3}\right): \delta 1.22\left(3 \mathrm{H}, \mathrm{t}, J=7.1 \mathrm{~Hz}, \mathrm{CH}_{3}\right), 2.79(2 \mathrm{H}, \mathrm{t}, J$ $\left.=5.7 \mathrm{~Hz}, \mathrm{O}=\mathrm{CCH}_{2}\right), 3.80\left(2 \mathrm{H}, \mathrm{t}, J=5.7 \mathrm{~Hz}, \mathrm{NCH}_{2}\right), 4.10\left(2 \mathrm{H}, \mathrm{q}, J=7.1 \mathrm{~Hz}, \mathrm{OCH}_{2}\right), 7.28(1 \mathrm{H}, \mathrm{s}$, $J=6.7 \mathrm{~Hz}, \mathrm{H}_{6}$ of 5-fluorouracil), $10.25(1 \mathrm{H}, \mathrm{s}, \mathrm{NH})$.

Ethyl 3-(6-amino-9H-purin-9-yl)propanoate (2a). Column chromathography on silica gel eluted with EtOAc/n-hexane (3/1) gave a colorless solid; mp 164-166 ${ }^{\circ} \mathrm{C}$ (Lit. ${ }^{9} \mathrm{mp} 167-168{ }^{\circ} \mathrm{C}$ ); ${ }^{1} \mathrm{H}$ NMR $\left(\mathrm{CDCl}_{3}\right): \delta 1.19\left(3 \mathrm{H}, \mathrm{t}, J=6.9 \mathrm{~Hz}, \mathrm{CH}_{3}\right), 2.95\left(2 \mathrm{H}, \mathrm{t}, J=5.9 \mathrm{~Hz}, \mathrm{O}=\mathrm{CCH}_{2}\right), 4.12(2 \mathrm{H}$, $\left.\mathrm{t}, J=6.9 \mathrm{~Hz}, \mathrm{OCH}_{2}\right), 4.49\left(2 \mathrm{H}, \mathrm{t}, J=5.9 \mathrm{~Hz}, \mathrm{NCH}_{2}\right), 6.41\left(\mathrm{~s}, 2 \mathrm{H}, \mathrm{NH}_{2}\right), 7.96\left(1 \mathrm{H}, \mathrm{s}, \mathrm{H}_{2}\right.$ of adenine), 8.45 (1H, s, $\mathrm{H}_{8}$ of adenine).

Butyl 3-(6-amino-9H-purin-9-yl)propanoate (2b). Column chromathography on silica gel eluted with EtOAc/n-hexane (3/1) gave a colorless solid; mp 135-137 ${ }^{\circ} \mathrm{C}$ (Lit. ${ }^{7} 134-136{ }^{\circ} \mathrm{C}$ ); ${ }^{1} \mathrm{H}$ NMR $\left(\mathrm{CDCl}_{3}\right): \delta 0.93\left(3 \mathrm{H}, \mathrm{t}, J=6.7 \mathrm{~Hz}, \mathrm{CH}_{3}\right), 1.36\left(2 \mathrm{H}, \mathrm{m}, \mathrm{CH}_{3} \mathrm{CH}_{2}\right), 1.58(2 \mathrm{H}, \mathrm{m}$, $\left.\mathrm{CH}_{3} \mathrm{CH}_{2} \mathrm{CH}_{2}\right), 2.93\left(2 \mathrm{H}, \mathrm{t}, J=5.8 \mathrm{~Hz}, \mathrm{O}=\mathrm{CCH}_{2}\right), 4.11\left(2 \mathrm{H}, \mathrm{t}, J=6.8 \mathrm{~Hz}, \mathrm{OCH}_{2}\right), 4.53(2 \mathrm{H}, \mathrm{t}, J=$ $\left.5.8 \mathrm{~Hz}, \mathrm{NCH}_{2}\right), 6.38\left(2 \mathrm{H}, \mathrm{s}, \mathrm{NH}_{2}\right), 7.99\left(1 \mathrm{H}, \mathrm{s}, \mathrm{H}_{2}\right.$ of adenine $), 8.40\left(1 \mathrm{H}, \mathrm{s}, \mathrm{H}_{8}\right.$ of adenine).

\section{2-Hydroxy-3-(2-methoxyphenoxy)propyl 3-(6-amino-9H-purin-9-yl)propanoate}

(2c). Column chromathography on silica gel eluted with EtOAc/n-hexane (3/1) gave a pale yellow oil; IR (neat): 3452, 3291, 3115, 3069, 2941, $1728 \mathrm{~cm}^{-1} ;{ }^{1} \mathrm{H}$ NMR $\left(\mathrm{CDCl}_{3}\right): \delta 2.96(2 \mathrm{H}, \mathrm{t}, J=5.8 \mathrm{~Hz}$, $\left.\mathrm{O}=\mathrm{CCH}_{2}\right), 3.72\left(3 \mathrm{H}, \mathrm{s}, \mathrm{CH}_{3}\right) ; 3.92-4.03(3 \mathrm{H}, \mathrm{m}), 4.19-4.29(3 \mathrm{H}, \mathrm{m}), 4.46(2 \mathrm{H}, \mathrm{t}, J=5.8 \mathrm{~Hz}$, $\left.\mathrm{NCH}_{2}\right), 6.47\left(2 \mathrm{H}, \mathrm{s}, \mathrm{NH}_{2}\right), 6.68-6.77(4 \mathrm{H}, \mathrm{m}), 7.94\left(1 \mathrm{H}, \mathrm{s}, \mathrm{H}_{2}\right.$ of adenine $), 8.46\left(1 \mathrm{H}, \mathrm{s}, \mathrm{H}_{8}\right.$ of adenine); ${ }^{13} \mathrm{C}$ NMR $\left(\mathrm{CDCl}_{3}\right): \delta 39.4,46.9,56.3,64.9,68.6,71.0,113.1,114.8,120.2,121.9$, 122.7, 140.6, 146.3, 149.4, 150.3, 153.0, 155.8, 171.4; MS $(\mathrm{m} / \mathrm{z}): 387\left(\mathrm{M}^{+}\right)$; Anal. calcd. for $\mathrm{C}_{18} \mathrm{H}_{21} \mathrm{~N}_{5} \mathrm{O}_{5}$ : C, 55.81; H, 5.46; N, 18.08. Found: $\mathrm{C}, 55.98 ; \mathrm{H}, 5.22 ; \mathrm{N}, 18.21$.

Ethyl 3-(6-amino-9H-purin-9-yl)-2-methylpropanoate (2d). Column chromathography on silica gel eluted with EtOAc/n-hexane (3/1) gave a colorless solid; mp 133-135 ${ }^{\circ} \mathrm{C}$ (Lit. ${ }^{7} \mathrm{mp} 134-$ $\left.137{ }^{\circ} \mathrm{C}\right) ;{ }^{1} \mathrm{H} \mathrm{NMR}\left(\mathrm{CDCl}_{3}\right): \delta 1.12-1.21\left(6 \mathrm{H}, \mathrm{m}, \mathrm{CH}_{2} \mathrm{CH}_{3}\right.$ and $\left.\mathrm{CHCH}_{3}\right), 3.13(1 \mathrm{H}, \mathrm{m}, \mathrm{O}=\mathrm{CCH})$, $4.09\left(2 \mathrm{H}, \mathrm{q}, J=6.9 \mathrm{~Hz}, \mathrm{OCH}_{2}\right), 4.22\left(1 \mathrm{H}, \mathrm{m}\right.$, one $\mathrm{H}$ of $\left.\mathrm{NCH}_{2}\right), 4.43\left(1 \mathrm{H}, \mathrm{m}\right.$, one $\mathrm{H}$ of $\left.\mathrm{NCH}_{2}\right)$, $6.24\left(2 \mathrm{H}, \mathrm{s}, \mathrm{NH}_{2}\right), 7.83\left(1 \mathrm{H}, \mathrm{s}, \mathrm{H}_{2}\right.$ of adenine $), 8.35\left(1 \mathrm{H}, \mathrm{s}, \mathrm{H}_{8}\right.$ of adenine).

Ethyl 3-(6-amino-9H-purin-9-yl)butanoate (2e). Column chromathography on silica gel eluted with EtOAc/n-hexane (3/1) gave a colorless solid; mp 101-103 ${ }^{\circ} \mathrm{C}$ (Lit. ${ }^{7} \mathrm{mp} 100-101{ }^{\circ} \mathrm{C}$ ); ${ }^{1} \mathrm{H}$ $\operatorname{NMR}\left(\mathrm{CDCl}_{3}\right): \delta 1.14\left(3 \mathrm{H}, \mathrm{t}, J=6.8 \mathrm{~Hz}, \mathrm{CH}_{2} \mathrm{CH}_{3}\right), 1.66\left(3 \mathrm{H}, \mathrm{d}, J=4.7 \mathrm{~Hz}, \mathrm{CHCH}_{3}\right), 2.89(1 \mathrm{H}$, $\mathrm{m}$, one $\mathrm{H}$ of $\left.\mathrm{O}=\mathrm{CCH}_{2}\right), 3.15\left(1 \mathrm{H}, \mathrm{m}\right.$, one $\mathrm{H}$ of $\left.\mathrm{O}=\mathrm{CCH}_{2}\right), 4.06\left(2 \mathrm{H}, \mathrm{q}, \mathrm{J}=6.8 \mathrm{~Hz}, \mathrm{OCH}_{2}\right), 4.97$ $(1 \mathrm{H}, \mathrm{m}, \mathrm{NCH}), 6.31\left(2 \mathrm{H}, \mathrm{s}, \mathrm{NH}_{2}\right), 7.80\left(1 \mathrm{H}, \mathrm{s}, \mathrm{H}_{2}\right.$ of adenine), 8.27 (1H, s, $\mathrm{H}_{8}$ of adenine).

Butyl 3-(6-chloro-9H-purin-9-yl)propanoate (2f). Column chromathography on silica gel eluted with EtOAc/n-hexane (1/1) gave a colorless oil (Lit. ${ }^{6}$ oil); ${ }^{1} \mathrm{H}$ NMR $\left(\mathrm{CDCl}_{3}\right): \delta 0.90(3 \mathrm{H}$, t, $\left.J=6.7 \mathrm{~Hz}, \mathrm{CH}_{3}\right), 1.33\left(2 \mathrm{H}, \mathrm{m}, \mathrm{CH}_{3} \mathrm{CH}_{2}\right), 1.56\left(2 \mathrm{H}, \mathrm{m}, \mathrm{CH}_{3} \mathrm{CH}_{2} \mathrm{CH}_{2}\right), 2.95(2 \mathrm{H}, \mathrm{t}, J=5.8 \mathrm{~Hz}$, $\left.\mathrm{O}=\mathrm{CCH}_{2}\right), 4.09\left(2 \mathrm{H}, \mathrm{t}, J=6.9 \mathrm{~Hz}, \mathrm{OCH}_{2}\right), 4.61\left(2 \mathrm{H}, \mathrm{t}, J=5.8 \mathrm{~Hz}, \mathrm{NCH}_{2}\right), 8.52\left(1 \mathrm{H}, \mathrm{s}, \mathrm{H}_{8}\right.$ of 6chloropurine), 8.99 (1H, s, $\mathrm{H}_{2}$ of 6-chloropurine). 
Butyl 3-(6-chloro-9H-purin-7-yl)propanoate (3f). Column chromathography on silica gel eluted with EtOAc/n-hexane (1/1) gave a pale yellow solid; mp 59-61 ${ }^{\circ} \mathrm{C}\left(\right.$ Lit. $\left.^{6} \mathrm{mp} 60-62{ }^{\circ} \mathrm{C}\right) ;{ }^{1} \mathrm{H}$ NMR $\left(\mathrm{CDCl}_{3}\right): \delta 0.93\left(3 \mathrm{H}, \mathrm{t}, J=6.8 \mathrm{~Hz}, \mathrm{CH}_{3}\right), 1.35\left(2 \mathrm{H}, \mathrm{m}, \mathrm{CH}_{3} \mathrm{CH}_{2}\right), 1.60(2 \mathrm{H}, \mathrm{m}$, $\left.\mathrm{CH}_{3} \mathrm{CH}_{2} \mathrm{CH}_{2}\right), 2.99\left(2 \mathrm{H}, \mathrm{t}, J=5.8 \mathrm{~Hz}, \mathrm{O}=\mathrm{CCH}_{2}\right), 4.11\left(2 \mathrm{H}, \mathrm{t}, J=6.7 \mathrm{~Hz}, \mathrm{OCH}_{2}\right), 4.87(2 \mathrm{H}, \mathrm{t}, J=$ $\left.5.8 \mathrm{~Hz}, \mathrm{NCH}_{2}\right), 8.61\left(1 \mathrm{H}, \mathrm{s}, \mathrm{H}_{8}\right.$ of 6-chloropurine), $9.09\left(1 \mathrm{H}, \mathrm{s}, \mathrm{H}_{2}\right.$ of 6-chloropurine).

Butyl 3-(6-0xo-1,6-dihydropurin-9-yl)propanoate (2g). Column chromathography on silica gel eluted with EtOAc/n-hexane (3/1) gave a colorless solid; mp 83-85 ${ }^{\circ} \mathrm{C}$ (Lit. ${ }^{6} \mathrm{mp} \mathrm{83-85}{ }^{\circ} \mathrm{C}$ ); ${ }^{1} \mathrm{H}$ NMR $\left(\mathrm{CDCl}_{3}\right): \delta 0.92\left(3 \mathrm{H}, \mathrm{t}, J=6.8 \mathrm{~Hz}, \mathrm{CH}_{3}\right), 1.35\left(2 \mathrm{H}, \mathrm{m}, \mathrm{CH}_{3} \mathrm{CH}_{2}\right), 1.58(2 \mathrm{H}, \mathrm{m}$, $\left.\mathrm{CH}_{3} \mathrm{CH}_{2} \mathrm{CH}_{2}\right), 2.87\left(2 \mathrm{H}, \mathrm{t}, J=5.8 \mathrm{~Hz}, \mathrm{O}=\mathrm{CCH}_{2}\right), 4.12\left(2 \mathrm{H}, \mathrm{t}, J=6.9 \mathrm{~Hz}, \mathrm{OCH}_{2}\right), 4.52(2 \mathrm{H}, \mathrm{t}, J=$ $\left.5.8 \mathrm{~Hz}, \mathrm{NCH}_{2}\right), 7.99\left(1 \mathrm{H}, \mathrm{s}, \mathrm{H}_{8}\right.$ of hypoxanthine), $8.56\left(1 \mathrm{H}, \mathrm{s}, \mathrm{H}_{2}\right.$ of hypoxanthine $), 10.43(1 \mathrm{H}, \mathrm{s}$, $\mathrm{NH})$.

Butyl 3-(6-oxo-1,6-dihydropurin-7-yl)propanoate (3g). Column chromathography on silica gel eluted with EtOAc/n-hexane (3/1) gave a colorless solid; mp 100-103 ${ }^{\circ} \mathrm{C}$ (Lit. ${ }^{6} \mathrm{mp} 99-102{ }^{\circ} \mathrm{C}$ ); ${ }^{1} \mathrm{H}$ NMR $\left(\mathrm{CDCl}_{3}\right): \delta 0.90\left(3 \mathrm{H}, \mathrm{t}, J=6.8 \mathrm{~Hz}, \mathrm{CH}_{3}\right), 1.38\left(2 \mathrm{H}, \mathrm{m}, \mathrm{CH}_{3} \mathrm{CH}_{2}\right), 1.59(2 \mathrm{H}, \mathrm{m}$, $\left.\mathrm{CH}_{3} \mathrm{CH}_{2} \mathrm{CH}_{2}\right), 2.90\left(2 \mathrm{H}, \mathrm{t}, J=5.9 \mathrm{~Hz}, \mathrm{O}=\mathrm{CCH}_{2}\right), 4.08\left(2 \mathrm{H}, \mathrm{t}, J=6.8 \mathrm{~Hz}, \mathrm{OCH}_{2}\right), 4.74(2 \mathrm{H}, \mathrm{t}, J=$ $\left.5.9 \mathrm{~Hz}, \mathrm{NCH}_{2}\right), 8.10\left(1 \mathrm{H}, \mathrm{s}, \mathrm{H}_{8}\right.$ of hypoxanthine), $8.64\left(1 \mathrm{H}, \mathrm{s}, \mathrm{H}_{2}\right.$ of hypoxanthine $), 10.48(1 \mathrm{H}, \mathrm{s}$, $\mathrm{NH})$.

\section{Acknowledgements}

The authors thank Payame Noor University and Persian Gulf Research Councils for the financial support of this work.

\section{References}

1. (a) Mikami, K. Green Reaction Media in Organic Synthesis, Blackwell Publishing, UK, Oxford, 2005. (b) Wasserscheid, P.; Welton, T. Ionic Liquids in Synthesis, Wiley-VCH, Weinheim, 2002. (c) Rogers, R. D. Ionic Liquids as Green Solvents: Progress and Prospects, Publisher: An American Chemical Society Publication, 2005. (d) Sarda, S. R.; Pathan, M. Y.; Paike, V. V.; Pachmase, P. R.; Jadhav, W. N.; Pawar, R. P. ARKIVOC 2006, (xvi), 43. (e) Legeay, J. C.; Eynde, J. J. V.; Toupet, L.; Bazureau, J. P. ARKIVOC 2007, (iii), 13. (f) Potewar, T. M.; Ingale, S. A.; Srinivasan, K. V. ARKIVOC 2008, (xiv), 100. (g) Vallette, H.; Ferron, L.; Coquerel, G.; Guillen, F.; Plaquevent, J.-C. ARKIVOC 2006, (iv), 200. (h) Mojtahedi, M. M.; Abaee, M. S.; Abbasi, H. J. Iran. Chem. Soc. 2006, 3, 93. (i) Dabiri, M.; Salehi, P.; Baghbanzadeh, M.; Shakouri, M.; Otokesh, S.; Ekrami, T.; Doosti, R. J. Iran. Chem. Soc. 2007, 4, 393.

2. De Clercq, E. Advance in Antiviral Drug Design, Vol. 1, Johnsson, N. G., Ed., Jai: Greenwich, 1993. 
3. (a) Thomas, G. Medicinal Chemistry, John Wiley: New York, 2000. (b) Doerge, R. F. Wilson and Gisvold's textbook of organic medicinal and pharmaceutical chemistry, $8^{\text {th }}$ Ed., J. B. Lippincott Company, 1982.

4. Taylor, E. C.; Sowinski, F. J. Am. Chem. Soc. 1969, 91, 2143.

5. Kelley, J. L.; Bullock, R. M.; Krochmal, M. P.; McLean, E. W.; Linn, J. A.; Durcan, M. J.; Cooper, B. R. J. Med. Chem. 1997, 40, 3207.

6. Zare, A.; Hasaninejad, A.; Beyzavi, M. H.; Parhami, A.; Moosavi Zare, A. R.; KhalafiNezhad, A.; Sharghi, H. Can. J. Chem. 2008, 86, 317.

7. Khalafi-Nezhad, A.; Zarea, A.; Soltani Rad, M. N.; Mokhtari, B.; Parhami, A. Synthesis 2005, 419.

8. Lazrek, H. B.; Khaider, H.; Rochdi, A.; Barascut, J.-L.; Imbach, J.-L. Tetrahedron Lett. 1996, 37, 4701.

9. Lira, E. P.; Huffman, C. W. J. Org. Chem. 1966, 31, 2188.

10. Gimbert, C.; Moreno-Maňas, M.; Pérez, E.; Vallribera, A. Tetrahedron 2007, 63, 8305.

11. Cai, Y.; Sun, X.-F.; Wang, N.; Lin, X.-F. Synthesis 2004, 671.

12. Xu, J.-M.; Qian, C.; Liu, B.-K.; Wu, Q.; Lin, X.-F. Tetrahedron 2007, 63, 986.

13. (a) Lazrek, H. B.; Rochdi, A.; Khaider, H.; Barascut, J.-L.; Imbach, J.-L.; Balzarini, J.; Witvrouw, M.; Pannecouque, C.; De Clercq, E. Tetrahedron 1998, 54, 3807. (b) Lazrek, H. B.; Khaider, H.; Rochdi, A.; Barascut, J.-L.; Imbach, J.-L. Nucleosides \& Nucleotides 1994, $13,811$.

14. Khalafi-Nezhad, A.; Zare, A.; Parhami, A.; Soltani Rad, M. N. ARKIVOC 2006, (xii), 161.

15. Khalafi-Nezhad, A.; Zare, A.; Parhami, A.; Soltani Rad, M. N.; Nejabat, G. R. Can. J. Chem. 2006, 84, 979.

16. Kotha, S.; Singh, K. Tetrahedron Lett. 2004, 45, 9607.

17. Chu, F.; Dueno, E. E.; Jung, K. W. Tetrahedron Lett. 1999, 40, 1847.

18. Churruca, F.; SanMartin, R.; Tellitu, I.; Domínguez, E. Tetrahedron Lett. 2003, 44, 5925.

19. Salvatore, R. N.; Smith, R. A.; Nischwitz, A. K.; Gavin, T. Tetrahedron Lett. 2005, 46, 8931.

20. Geraci, C.; Piattelli, M.; Neri, P. Tetrahedron Lett. 1996, 37, 3899.

21. Ubukata, M.; Isono, K. Tetrahedron Lett. 1986, 27, 3907.

22. (a) Loupy, A. Microwave in Organic Synthesis, Wiley-VCH, Weinheim, 2006. (b) Varma, R. S. Advances in Green Chemistry: Chemical Synthesis Using Microwave Irradiation, Astra Zeneca Research Foundation, Kavitha Printers: Bangalore, India, 2002. (c) Tanka, K. Solvent Free Organic Synthesis, Wiley-VCH, Weinhein, 2003. (d) Dewan, S. K.; Singh. R.; Kumar, A. ARKIVOC 2006, (ii), 41. (e) Zou, X.; Tu, S.; Shi, F.; Xu, J. ARKIVOC 2006, (ii), 130. (f) Reddy, P. B.; Singh, P. P.; Sawant, S. D.; Koul, S.; Taneja, S. C.; Kumar, H. M. S. ARKIVOC 2006, (xiii), 142. (g) Nadaraj, V.; Selvi, S. T.; Sasi, R. ARKIVOC 2006, (v), 82. (h) Rajithaa, B.; Kumara, V. N.; Someshwara, P.; Madhava, J. V.; Reddy, P. N.; Reddyb, Y. T. ARKIVOC 2006, (xii), 23. (i) Mojtahedi, M. M.; Ghasemi, M. H.; Abaee, M. S.; Bolourtchian, M. ARKIVOC 2005, (xv), 68. (j) Rábarová, E.; Koišs, P.; Lácová, M.; Krutošíková, A. ARKIVOC 2004, (i), 110. (k) Lukács, G.; Porcs-Makkay, M.; Komáromi, A.; 
Simig, G. ARKIVOC 2008, (iii), 17. (1) Nichols, C. E.; Youssef, D.; Harris, R. G.; Jha, A. ARKIVOC 2006, (xiii), 64. (m) El Ashry, E. S. H.; Awada, L. F.; Ibrahim, E. S. I.; Bdeewy, O. K. ARKIVOC 2006, (ii), 178. (n) Azizian, J.; Mohammadizadeh, M. R.; Zomorodbakhsh, S.; Mohammadi, A. A.; Karimi, A. R. ARKIVOC 2007, (xv), 24. (o) Gupta, M.; Wakhloo, B. P. ARKIVOC 2007, (i), 94. (p) Khalafi-Nezhad, A.; Zare, A.; Parhami, A.; Soltani Rad, M. N.; Nejabat, G. R. J. Iran. Chem. Soc. 2007, 4, 271. (q) Imanzadeh, G. H.; Khalafi-Nezhad, A.; Zare, A.; Hasaninejad, A.; Moosavi Zare, A. R.; Parhami, A. J. Iran. Chem. Soc. 2007, 4, 229. (r) Desai, K. G.; Raval, J. P.; Desai, K. R. J. Iran. Chem. Soc. 2006, 3, 233.

23. (a) Soltani Rad, M. N.; Khalafi-Nezhad, A.; Behrouz, S.; Faghihi, M. A.; Zare, A.; Parhami, A. Tetrahedron 2008, 64, 1778. (b) Khalafi-Nezhad, A.; Parhami, A.; Soltani Rad, M. N.; Zolfigol, M. A.; Zare A. Tetrahedron Lett. 2007, 48, 5219. 